\title{
DEVELOPMENT OF POLYMER MODIFIED GRAPHENE OXIDE NANOCOMPOSITE MEMBRANES TO REDUCE BOTH SCALING AND BIOFOULING
}

Mohammad Y. Ashfaq ${ }^{1 *}$, Mohammad A. Al-Ghouti ${ }^{1}$, Hazim Qiblawey ${ }^{2}$, Nabil Zouari ${ }^{1}$ 1Department of Biological and Environmental Sciences, College of Arts and Science, Qatar University, State of Qatar - Doha. P.O. Box: 2713, Email: ma1203537@qu.edu.qa 2Departmet of Chemical Engineering, Collage of Engineering, Qatar University, State of Qatar - Doha. P.O. Box: 2713

\section{ABSTRACT}

in scaling is one of the major issues affecting its widespread application in the desalination industry.

In this research, the effect of concentration of calcium and sulfate ions from 20 to $150 \mathrm{mM}$ and temperature from 5 to $35^{\circ} \mathrm{C}$ on calcium sulfate scaling of reverse asmosis (RO) and Graphene oxide functionalized RO membranes was investigated.

It was found that increase of concentration as well as temperature enhances the mineral scaling, where morphology of crystals varies from rod shaped to rosette structures.

It was also observed that commonly found seawater bacteria can use antiscalants as an energy/carbon source thereby degrading them and reducing their efficiency to reduce mineral scaling.

Moreover, bacteria were found to be capable of inducing/mediating calcium sulfate precipitation on RO membranes further enhancing the mineral scaling. Therefore, it was important to modify RO membranes capable of simultaneously reduce both mineral scaling and biofouling.

For this purpose, RO membrane was modified with antibacterial graphene oxide and polymer anliscalants using microwave radiation technique.

It was found that the modified membranes were able to inhibit microbial growth up to $95 \%$, while, mineral scaling was also reduced by $97 \%$.

Hence, it was concluded that the coating of polymer modified graphene oxide anocomposites on RO membranes can simultaneously reduce both biofouling and scaling.

So far, such dual

\section{INTRODUCTION}

Al-Ghouti's research team has recently used Drivers-Pressures-StateImpact-Response (DPSIR) framework to analyze water resources in Qatar (Ashfaq et al., 2018) and it was found that the unsustainable consumption of water resources in both domestic and industrial sectors are pressurizing the Oatar's limited water resources. Sea water Reverse Osmosis is an environmentally and economically viable technique to cope with rising water demands in Qatar. However, membrane fouling (scaling and biofouling) is affecting its larever, membrane fouling (scaling and biofoun

The-scale application in desalmation industry. Therefore, it is important to develop in-depth understanding of interaction between biofoulants and scalants and develop RO membranes capable of reducing both type of fouling.

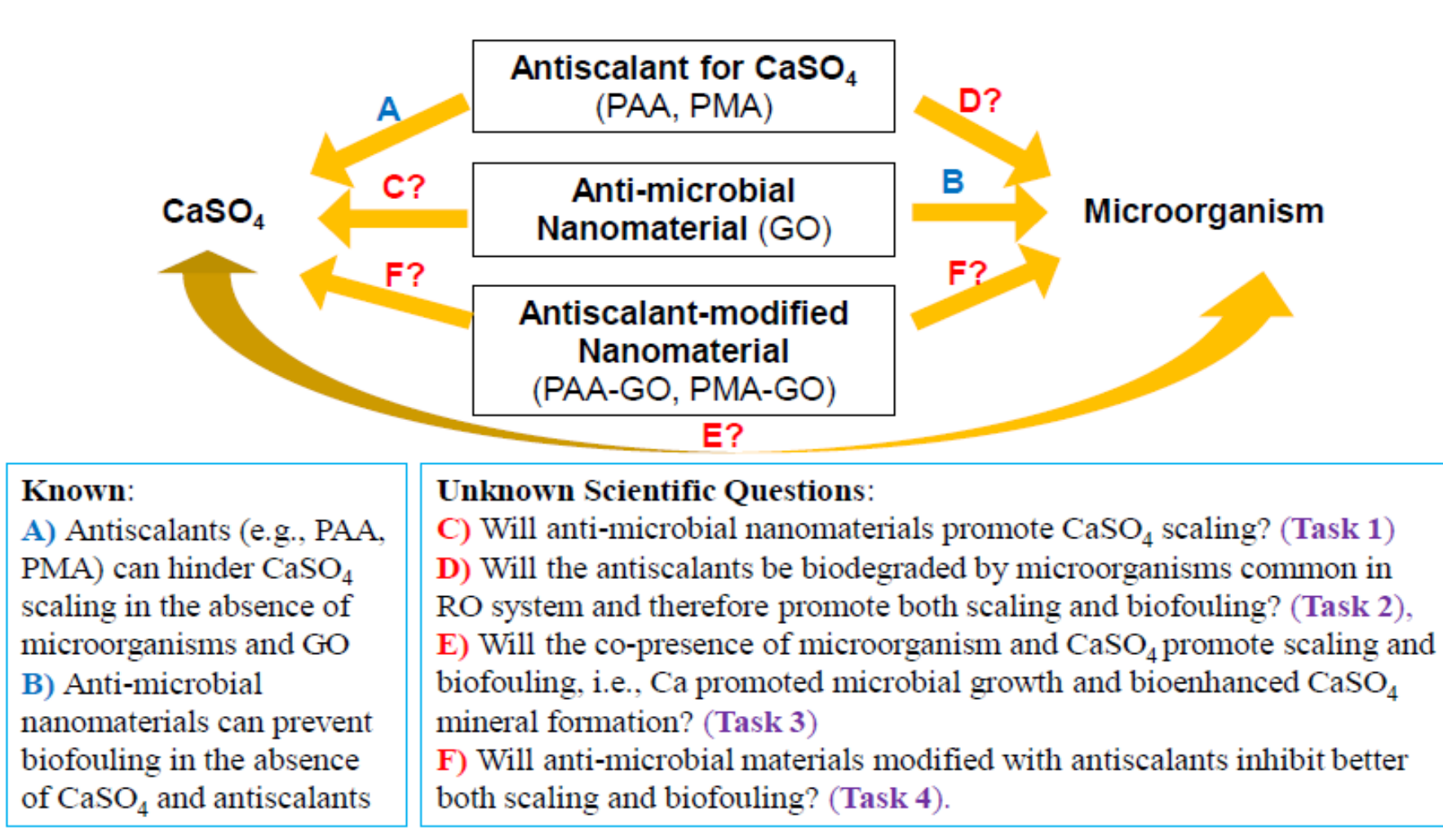

Objective 1: Understand effects and mechanisms of anti-microbial nanomaterials (GO) on $\mathrm{CaSO}_{4}$ scaling.

Objective 2: Demonstrate and determine biodegradation of polymer antiscalants used in RO systems.

Objective 3: Investigate the interactions between microorganisms and $\mathrm{CaSO}_{4}$ in $\mathrm{RO}$ system.

Objective 4: Determine whether new coatings (GO-PAA, GO-PMA) will prevent biofouling and scaling.

\section{METHODOLOGY}

The effect of temperature and concentration was studied using membrane filtration setup developed in Lab.

Seawater bacteria were isolated using special media and were identified using MALDI-TOF MS and were investigated for antiscalant degradation using modified growth medium

Similarly, the microorganisms-mineral interactions were studied in specially prepared medium in the presence of RO membranes.

For characterization, SEM-EDX, FTIR, and XRD techniques were used.

The membrane surface was modified with polymer modified graphene oxide nanocomposites and their antibiofouling and antiscaling potential was investigated.

\section{RESULTS and DISCUSSION}

3.1.1 Calcium Sulfate Scaling (Effect of Ca concentration): The results of scanning electron microscopy (SEM) showed that the morphology of crystals varied with the concentration from rod shaped to broad rosette structures (Fig. 02)

- It was also found that the membrane surface was fully covered with precipitates which resulted from both bulk and surface crystallization at higher concentration of calcium ions.

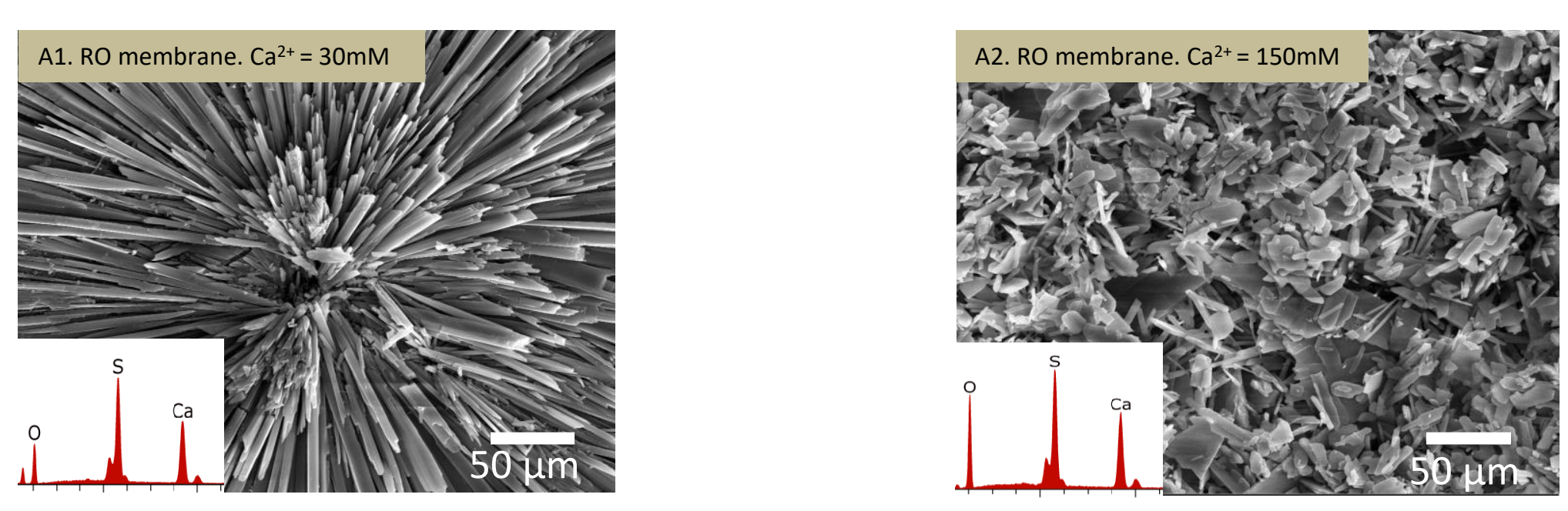

Figure 02: SEM - EDS analysis of precipitates on RO membran

3.1.2 Calcium Sulfate Scaling (Effect of Temperature)

- The results of scanning electron microscopy (SEM) showed that the morphology of crystals varied with the concentration from rod shaped to broad rosette structures (Fig. 05)

It was also found that the membrane surface was fully covered with precipitates which resulted from both bulk and surface crystallization at higher temperature.
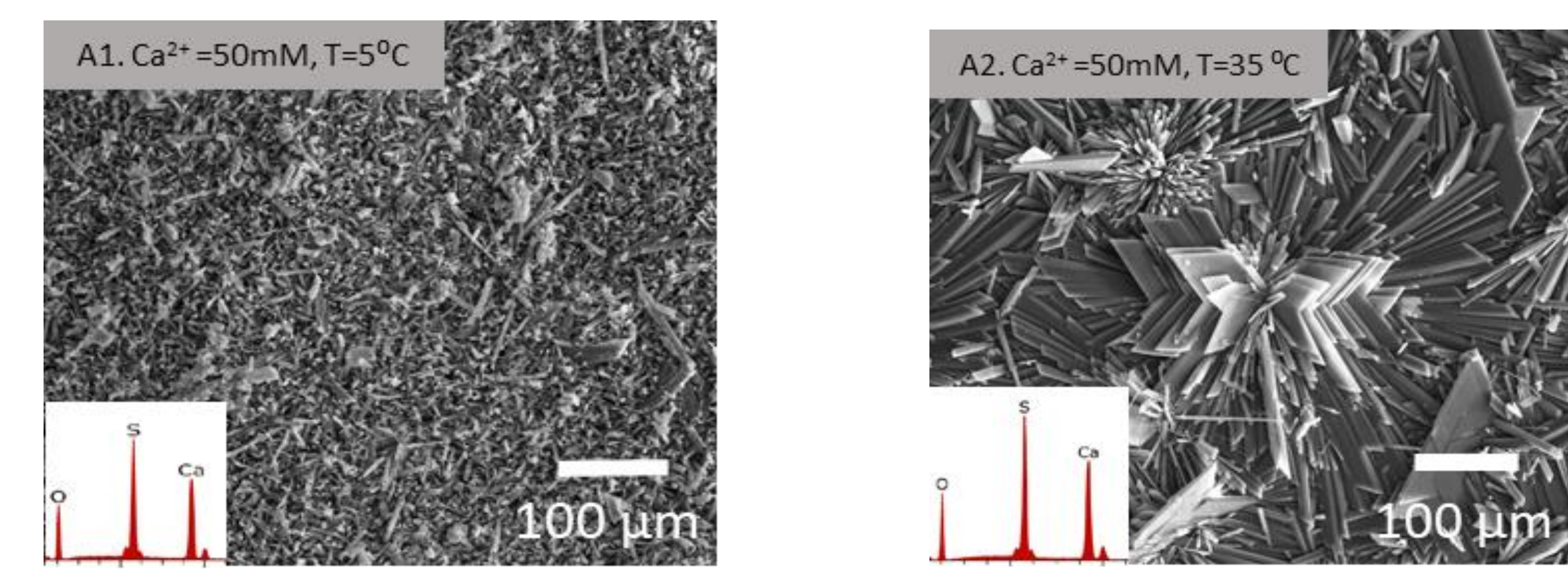

Figure 05: SEM - EDS analysis of precipitates on RO membran

3.2. Interaction between Microorganisms and Antiscalants:

Several strains were previously isolated from Qatar's seawater and were identified and differentiated using MALDI-TOF MS (Ashfaq et al., 2019a). Some identified strains included $H$. aquamarina, $H$. elongata, P. fragi and P. stutzeri among others.

The results of biodegradation showed that these microorganisms can use antiscalants as a source of carbon and energy, which may lead to the enhanced biofouling and scaling (Fig. 10) (Ashfaq et al., 2019b).

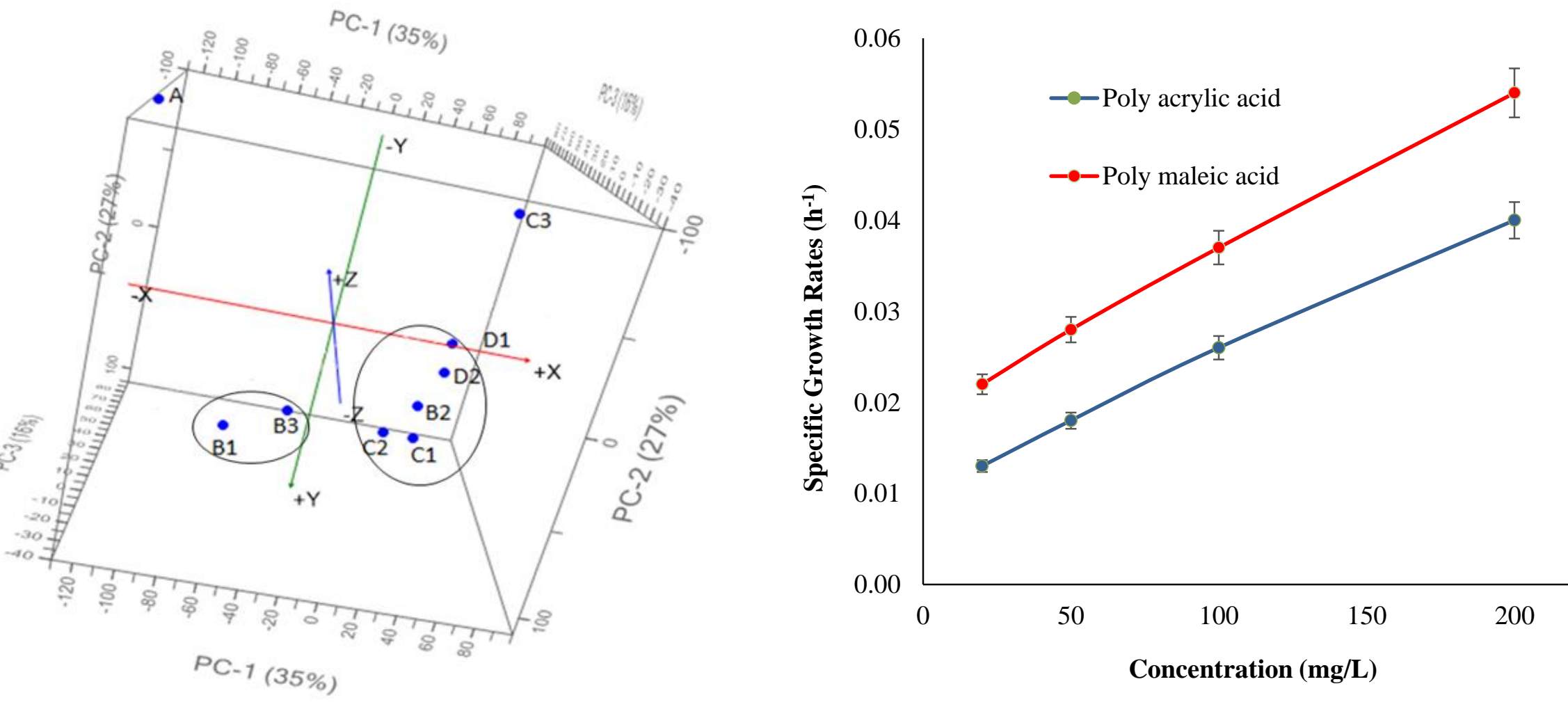

Figure 07: (a) Principle component analysis of isolated $H$. aquamarina strains,

(b) Specific growth rates of $H$. aquamarina strain in antiscalant containing medium

\subsection{Interaction between Microorganisms and $\mathrm{CaSO}_{4}$ scaling:}

Fig. 11 shows the $\mathrm{CaSO}_{4}$ precipitated in the liquid medium and on the RO membrane and

Results showed that the selected strains of $H$. aquamarina and P. fragi have the ability to form $\mathrm{CaSO}_{4}$ minerals in $\mathrm{RO}$ membrane systems causing increase in membrane scaling.
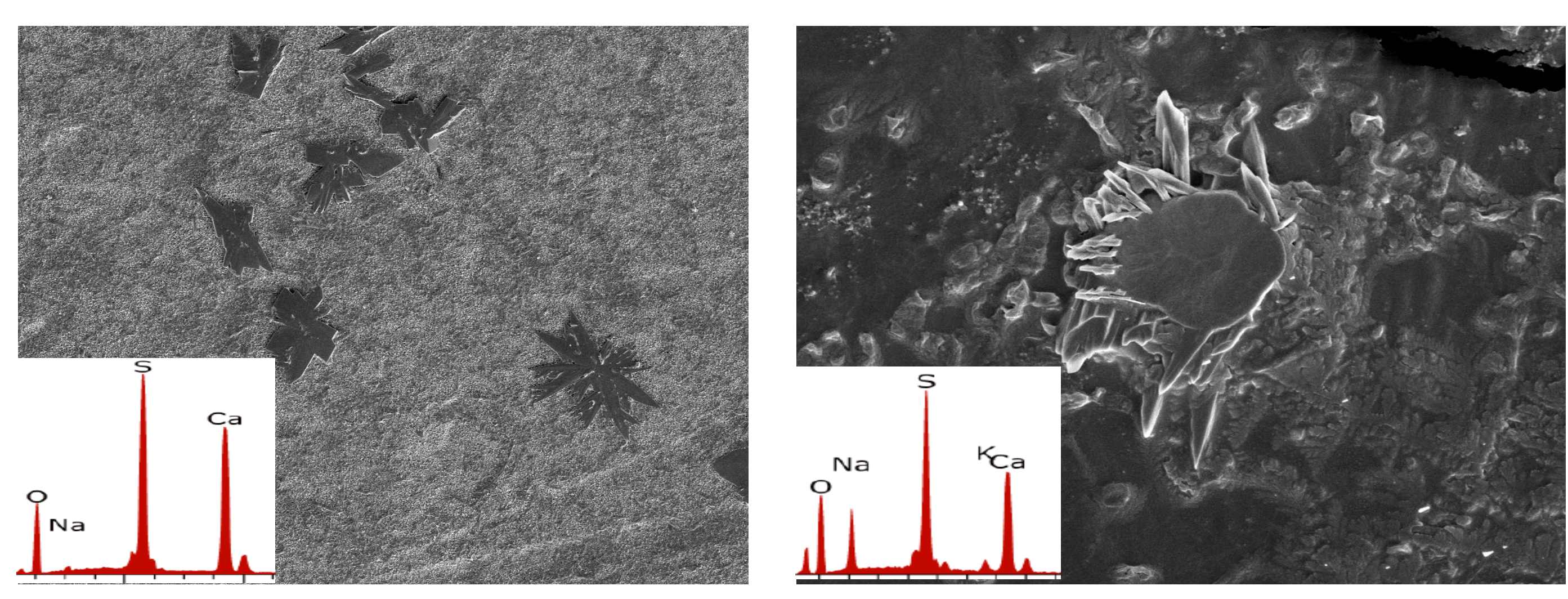

in the presence of $H$. aquamarina.
3.4. Preparation of Modified RO membranes

RO membrane was modified with antibacterial graphene oxide and polymer antiscalants using microwave radiation technique.

- It was found that the modified membranes were able to inhibit microbial growth up to $95 \%$, while, mineral scaling was also reduced by $97 \%$.

Hence, it was concluded that the coating of polymer modified graphene oxide nanocomposites on RO membranes can simultaneously reduce both biofouling and scaling

- So far, such dual characteristics of modified membranes have not been reported in the literature.

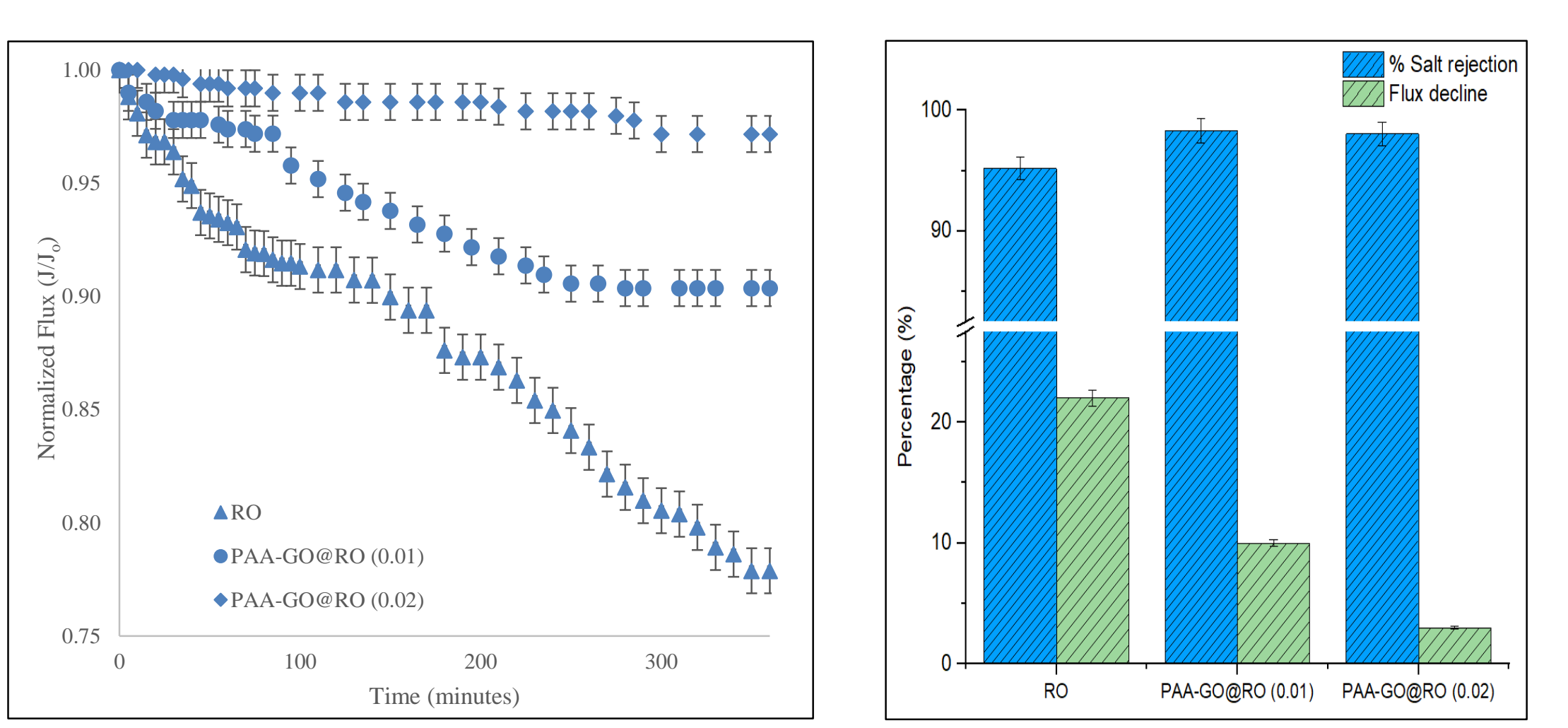

Fig. 6. (a) Decline of normalized flux with time; (b) Comparison of \%salt rejection and \% flux decline between RO and GO@RO membranes
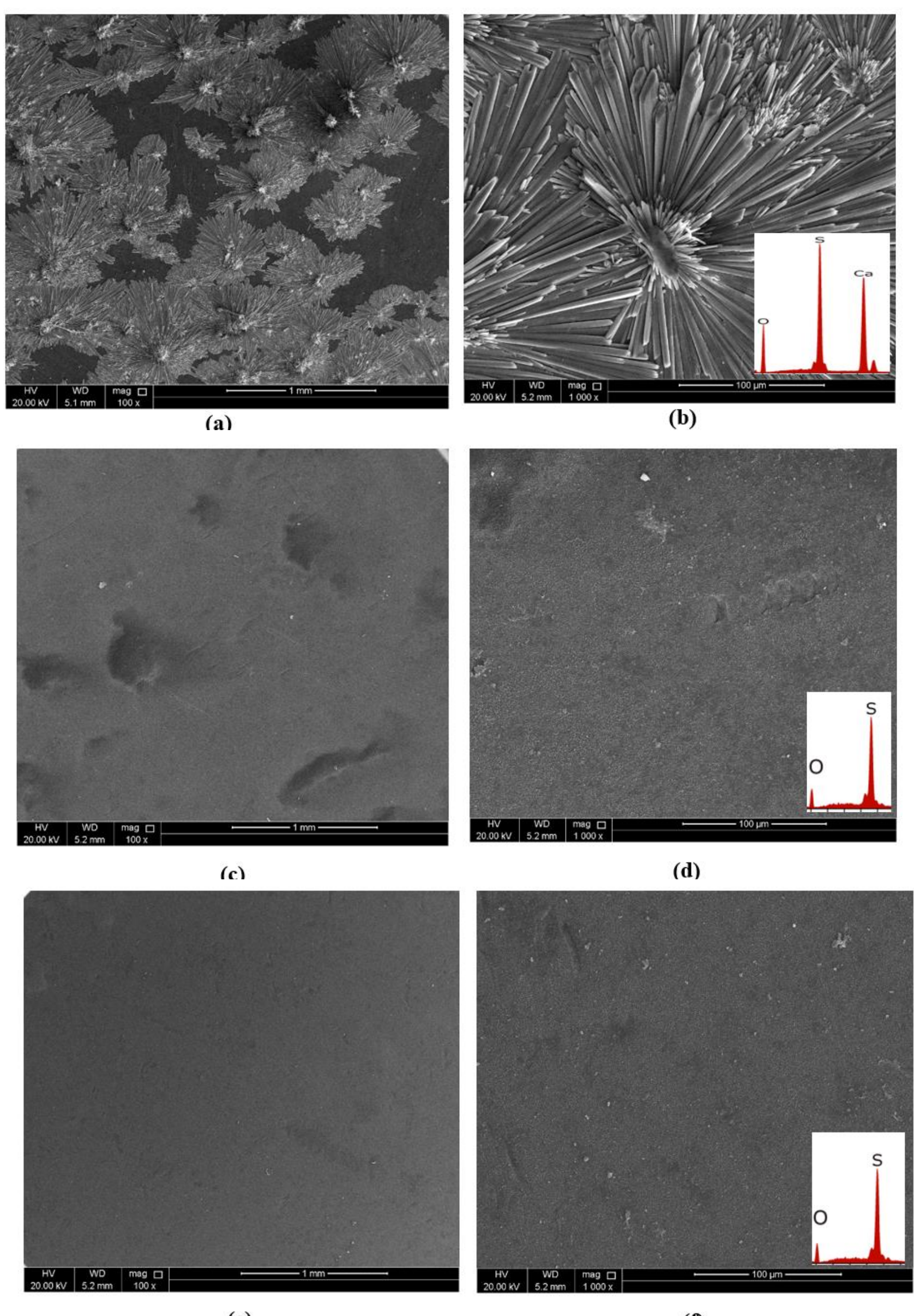

Fig. 7. SEM-EDX analysis of the scaled membrane (a, b) RO; (c, d) PAA-GO@RO (0.01); (e, f) PAA-GO@RO (0.02)

\section{CONCLUSION \& RECOMENDATION}

It was found that the increase in calcium ions enhanced the membrane scaling as shown by the increased membrane surface coverage by crystals, and intense flux decline over time at higher concentrations.

- The research also concludes the use of antiscalants in SWRO can potentially increase the risk of biofouling, thereby, reducing their ability to reduce scaling due to their biodegradation.

In addition, it was also found that these microorganisms have the capability to precipitate calcium sulfate in the solution as well as on the membrane.

- The functionalization of RO membrane surface with GO helped to improve membrane surface properties in terms of surface smoothness and hydrophilicity.

- Hence, it was concluded that the coating of polymer modified graphene oxide nanocomposites on RO membranes can simultaneously reduce both biofouling and scaling.

Acknowledgement: This research was made possible by NPRP grant \# [9-3181-064] from the Qatar National Research Fund (a member of Qatar Foundation). The findings achieved herein are solely the responsibility of the author[s].

\section{References:}

1. M.Y. Ashfaq, M.A. Al-Ghouti, H. Qiblawey, N. Zouari, Sci. Tota Environ. 656 (2019a) 910-920.

2. M.Y. Ashfaq, M.A. Al-Ghouti, H. Qiblawey, N. Zouari, Environ. Prog. Sustain. Energy 0, (2018).

3. M.Y. Ashfaq, M.A. Al-Ghouti, H. Qiblawey, N. Zouari, J. Biofouling $0,(2019 b)$

4. M.Y. Ashfaq, M.A. Al-Ghouti, D.A. Da'na, H. Qiblawey, N. Zouari' Sci. Total Environ. 703 (2020) 134726. 